\title{
Regret Aversion, Regret Neutrality, and Risk Aversion in Production
}

\section{Xu GUO and Wing-Keung WONG}

\author{
2017
}

EGC Report No: 2017/09

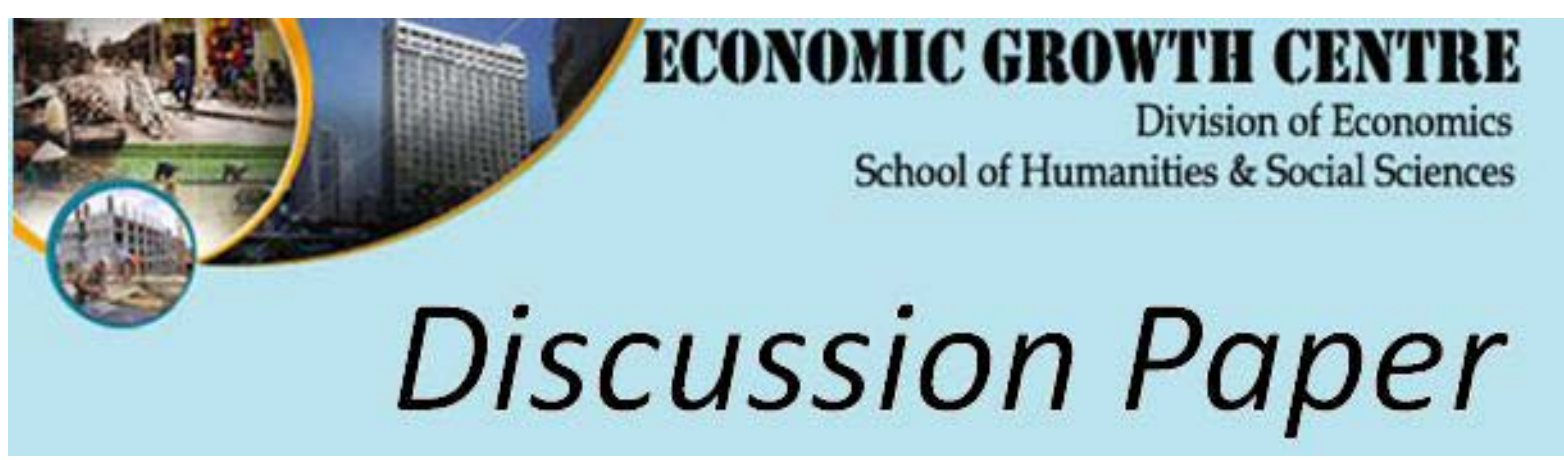


The author(s) bear sole responsibility for this paper.

Views expressed in this paper are those of the author(s) and not necessarily those of the Economic Growth Centre, NTU. 


\title{
Regret Aversion, Regret Neutrality, and Risk Aversion in Production
}

\author{
$\mathrm{Xu} \mathrm{Guo}^{1}$ and Wing-Keung Wong ${ }^{2,3,4 *}$ \\ ${ }^{1}$ School of Statistics, Beijing Normal University, Beijing \\ ${ }^{2}$ Department of Finance and Big Data Research Center, Asia University \\ ${ }^{3}$ Department of Economics and Finance, Hang Seng Management College \\ ${ }^{4}$ Department of Economics, Lingnan University
}

\begin{abstract}
Previous studies focus on the comparison of the optimal output levels of regret-averse firms under uncertainty and firms under certainty. This paper extends the theory by investigating the effects of both regret-aversion and regret-neutrality on production. We compare the optimal output levels of both regret-averse and regret-neutral firms with purely risk-averse firms under uncertainty and firms under certainty. We first show that the regret-neutral firms will surely produce more than its purely risk-averse counterparts and surely produce less than firms under certainty. Thereafter, we give sufficient conditions to ensure the regret-averse firms to produce more than both purely risk-averse and regret-neutral counterparts and study the comparative statics of the optimal production. We also develop properties of regretaversion on production in a binary model. The finding in this paper is useful for production managers in their decision on the production.
\end{abstract}

Keywords: Production; Regret aversion; Regret neutrality; Risk aversion; Regret theory; Uncertainty.

JEL Classification : D21, D24, D81.

\footnotetext{
*Corresponding author: Wing-Keung Wong; Email: wong@asia.edu.tw. The second author would like to thank Professors Robert B. Miller and Howard E. Thompson for their continuous guidance and encouragement. This research has been partially supported by the Fundamental Research Funds for the Central Universities, China Postdoctoral Science Foundation (2017M610058) and Natural Science Foundation of China NSFC (11626130, 11601227), Beijing Normal University, Asia University, Hang Seng Management College, Lingnan University, Hong Kong Baptist University and the Research Grants Council (RGC) of Hong Kong (project numbers 12502814 and 12500915), and Ministry of Science and Technology (MOST), R.O.C. This research is done when the second author is visiting Economic Growth Centre, School of Social Sciences, Nanyang Technological University.
} 


\section{Introduction}

Regret occurs when the ex-ante optimal decisions turn out to be ex-post suboptimal. This kind of behavioral characteristic is common and supported by our life experience and experimental literature (Loomes, 1988; Loomes and Sugden, 1987; Starmer and Sugden, 1993). To formally analyze this natural regret-averse preference, Bell (1982) and Loomes and Sugden (1982) introduce the regret theory that formally defines regret as the disutility of not having made the ex-post optimal decision. The regret theory is then further axiomatized by Sugden (1993) and extended by Quiggin (1994).

In traditional economic analysis of competitive firms with uncertain output price s(Sandmo 1971; Broll 1992; Viaene and Zilcha 1998), many studies consider purely risk-averse firms without taking the regret-averse preferences into account. Recently, Wong (2014) investigates production behavior of a competitive firm under both riskaversion and also regret-aversion. For tractability, he adopts the additive separable function proposed by Braun and Muermann (2004) and Muermann et al. (2006). By doing so, Wong (2014) provides a sufficient condition under which the optimal output level of the regret-averse firm under uncertainty is less than that under certainty. Thereafter, several authors, for example, Niu et al. (2014), Egozcue et al. (2015), and Broll et al. (2016, 2017), obtain different sufficient conditions to assure the regretaverse firms to produce less than firms without uncertainty.

In this paper we extend the theory by investigating the effects of both regretaversion and regret-neutrality on production. We compare the optimal output levels of both regret-averse and regret-neutral firms with purely risk-averse firms under uncertainty and firms under certainty.

We first show that the regret-neutral firms will surely produce more than its purely risk-averse counterparts and surely produce less than firms under certainty. Thereafter, we give sufficient conditions to ensure the regret-averse firms to produce 
more than both purely risk-averse and regret-neutral counterparts. We also study the comparative statics of the optimal production such that the regret-averse firms will produce more when they are more regret-averse.

To give more insights, we consider a simple binary model wherein the uncertain output price can take on either a low value or a high value with positive probability. Using this binary model, we show that the regret-averse firm could optimally produce more than purely risk-averse firm, especially when the low output price is very likely to prevail. This weakens the conditions set in the general case for the regret-averse competitive firm and give us insight to set the conjectures that in general the regretaverse firms will produce more than risk-averse firms, and the more regret-averse they are, they will produce more. The findings in this paper provide valuable complements of existing results and are useful for production managers in their decision on the production.

The rest of this paper is organized as follows. Section 2 delineates the model and develop properties for the competitive firm under uncertainty when the firm's preferences exhibit not only risk-aversion but also regret-aversion or regret-neutrality. Section 3 further studies properties of regret-aversion on production in a binary model. The final section concludes.

\section{The Theory}

In this section, we delineate the model of the competitive firm under uncertainty when the firm's preferences exhibit not only risk-aversion but also regret-aversion or regret-neutrality. We provide sufficient conditions under which the optimal output levels of the regret-averse and regret-neutral firms are larger than that of risk-averse firm. We also study the comparative statics of the optimal output levels when the regret coefficient changes. 


\subsection{Model setting}

We first briefly introduce the model. Consider a competitive firm which produces a single commodity with uncertain output price $\tilde{P}$ and the output level $Q \geq 0$, according to a special cost function $C(Q)$ satisfying $C(0)=C^{\prime}(0)=0, C^{\prime}(\cdot)>0$, and $C^{\prime \prime}(\cdot)>0 .{ }^{1}$ The support of $\tilde{P}$ is $[\underline{P}, \bar{P}]$ with $0<\underline{P}<\bar{P}<\infty$ and the firm's final profit is given by $\tilde{\Pi}=\tilde{P} Q-C(Q)$. To account for the regret that ex-post suboptimal decision has been made, Wong (2014) introduces the following bivariate utility function: ${ }^{2}$

$$
V\left(\Pi, \Pi^{\max }-\Pi\right)=U(\Pi)-\beta G\left(\Pi^{\max }-\Pi\right)
$$

Here, $\Pi^{\max }$ is the maximum profit that the firm could have earned if the realized output price is known in advance. Furthermore, if we have observed the realized output price $P, \Pi^{\max }$ would take the form that $\Pi^{\max }(P)=P Q(P)-C[Q(P)]$ with $C^{\prime}[Q(P)]=P . U(\cdot)$ is a von Neumann-Morgenstern utility function with $U^{\prime}(\cdot)>0$ and $U^{\prime \prime}(\cdot)<0$, accounting for the firm's risk-aversion. While $G(\cdot)$ is a regret function such that $G(0)=0, G^{\prime}(\cdot) \geq 0$, and $G^{\prime \prime}(\cdot) \geq 0 .{ }^{3}$ The parameter $\beta \geq 0$ is a constant regret coefficient, indicating the extent of the regret-aversion.

As a result, the production decision problem of the competitive firm reads:

$$
\max _{Q \geq 0} E\left\{U[\Pi(\tilde{P})]-\beta G\left[\Pi^{\max }(\tilde{P})-\Pi(\tilde{P})\right]\right\}
$$

Here, $E(\cdot)$ is the expectation operator with respect to the cumulative distribution function, $F(P)$, of the random output price $\tilde{P}$.

\footnotetext{
${ }^{1}$ We follow Wong (2014) to assume the strict convexity property of the cost function. This assumption reflects the fact that the firm's production technology exhibits decreasing return to scale.

${ }^{2}$ We follow Wong (2014) to use his notations. Braun and Muermann (2004) and Muermann et al. (2006) also use similar notations.

${ }^{3}$ Wong (2014) imposes this assumption to indicate that the more pleasurable the consequence that might have been, the more regret will be experienced. We modify the assumptions $G^{\prime}(\cdot)>0$ and $G^{\prime \prime}(\cdot)>0$ set by Wong $(2014)$ to $G^{\prime}(\cdot) \geq 0$, and $G^{\prime \prime}(\cdot) \geq 0$.
} 
The first-order condition is then given by:

$$
H\left(Q^{*}\right)=E\left\{\left\{U^{\prime}\left[\Pi^{*}(\tilde{P})\right]+\beta G^{\prime}\left[\Pi^{\max }(\tilde{P})-\Pi^{*}(\tilde{P})\right]\right\}\left[\tilde{P}-C^{\prime}\left(Q^{*}\right)\right]\right\}=0
$$

where an asterisk $\left({ }^{*}\right)$ indicates an optimal level. The second order condition is satisfied (Wong, 2014).

Since the seminal work of Wong (2014), several authors, for example, Niu et al. (2014), Egozcue et al. (2015), and Broll et al. (2016, 2017) compare the optimal output levels for regret-averse firms under uncertainty and under certainty. In this paper, we extend their study to compare the optimal output levels between the regret-averse and purely risk-averse firms.

To make comparison easier, we denote the optimal output level and optimal profit by $Q_{*}$ and $\Pi_{*}(\tilde{P})$, respectively, when the firm is purely risk-averse; that is, $\beta=0$. For $Q_{*}$, we have:

$$
E\left\{U^{\prime}\left[\Pi_{*}(\tilde{P})\right]\left[\tilde{P}-C^{\prime}\left(Q_{*}\right)\right]\right\}=0
$$

Since $U^{\prime}(\cdot)>0$, it is clear that

$$
E\left\{U^{\prime}\left[\Pi_{*}(\tilde{P})\right]\left[\underline{P}-C^{\prime}\left(Q_{*}\right)\right]\right\}<E\left\{U^{\prime}\left[\Pi_{*}(\tilde{P})\right]\left[\tilde{P}-C^{\prime}\left(Q_{*}\right)\right]\right\}=0<E\left\{U^{\prime}\left[\Pi_{*}(\tilde{P})\right]\left[\bar{P}-C^{\prime}\left(Q_{*}\right)\right]\right\}
$$

As a result, we get $\underline{P}-C^{\prime}\left(Q_{*}\right)<0<\bar{P}-C^{\prime}\left(Q_{*}\right)$.

Evaluating $H(Q)$ at $Q=Q_{*}$ and applying Equation (2.4), we get:

$$
\begin{aligned}
H\left(Q_{*}\right) & =\beta E\left\{G^{\prime}\left[\Pi^{\max }(\tilde{P})-\Pi_{*}(\tilde{P})\right]\left[\tilde{P}-C^{\prime}\left(Q_{*}\right)\right]\right\} \\
& =\beta \operatorname{Cov}\left(G^{\prime}\left[\Pi^{\max }(\tilde{P})-\Pi_{*}(\tilde{P})\right], \tilde{P}\right)+\beta E\left\{G^{\prime}\left[\Pi^{\max }(\tilde{P})-\Pi_{*}(\tilde{P})\right]\right\}\left[E(\tilde{P})-C^{\prime}\left(Q_{*}\right)\right] \\
& =\beta E\left\{G^{\prime}\left[\Pi^{\max }(\tilde{P})-\Pi_{*}(\tilde{P})\right] \tilde{P}\right\}-\beta C^{\prime}\left(Q_{*}\right) E\left\{G^{\prime}\left[\Pi^{\max }(\tilde{P})-\Pi_{*}(\tilde{P})\right]\right\} .
\end{aligned}
$$

Thus, as long as

$$
\frac{E\left\{G^{\prime}\left[\Pi^{\max }(\tilde{P})-\Pi_{*}(\tilde{P})\right] \tilde{P}\right\}}{E\left\{G^{\prime}\left[\Pi^{\max }(\tilde{P})-\Pi_{*}(\tilde{P})\right]\right\}} \geq C^{\prime}\left(Q_{*}\right),
$$


$H\left(Q_{*}\right) \geq 0$, and then, according to Equation (2.3) and the second order condition, we have $Q^{*} \geq Q_{*}$. To find out the meaning of the above condition clearly, we define the following function:

$$
\Phi(P)=\int_{\underline{P}}^{P} \frac{G^{\prime}\left[\Pi^{\max }(\tilde{P})-\Pi_{*}(\tilde{P})\right]}{E\left\{G^{\prime}\left[\Pi^{\max }(\tilde{P})-\Pi_{*}(\tilde{P})\right]\right\}} d F(P) \quad \text { for all } \quad P \in[\underline{P}, \bar{P}] .
$$

It is evident that $\Phi^{\prime}(P)>0, \Phi(\underline{P})=0$, and $\Phi(\bar{P})=1$. Thus, we can interpret $\Phi(P)$ as a cumulative distribution function of $\tilde{P}$. Hence, condition above can be also expressed as $E_{\Phi}(\tilde{P}) \geq C^{\prime}\left(Q_{*}\right)$; that is, as long as the transformed expectation of uncertain output price, $E_{\Phi}(\tilde{P})$, is larger than the marginal cost of production $C^{\prime}\left(Q_{*}\right)$, regret-averse firm will produce more than its purely risk-averse counterpart. We will discuss this issue more in the following subsections.

\subsection{Regret-neutral competitive firm}

Over here, we discuss whether the regret-neutral competitive firm will produce more than the purely risk-averse firm. To do so, we consider a special case with $G^{\prime}(\cdot) \equiv$ $m$, a constant. This corresponds to the regret-neutral firm. In this case, we get $\Phi(P)=F(P)$ and $E_{\Phi}(\tilde{P})=E(\tilde{P})$. The condition $E_{\Phi}(\tilde{P}) \geq C^{\prime}\left(Q_{*}\right)$ is then the same as $E(\tilde{P}) \geq C^{\prime}\left(Q_{*}\right)$. From Equation (2.4), we know that

$$
\operatorname{Cov}\left(U^{\prime}\left[\Pi_{*}(\tilde{P})\right], \tilde{P}\right)+E\left\{U^{\prime}\left[\Pi_{*}(\tilde{P})\right]\right\}\left[E(\tilde{P})-C^{\prime}\left(Q_{*}\right)\right]=0 .
$$

Since $\operatorname{Cov}\left(U^{\prime}\left[\Pi_{*}(\tilde{P})\right], \tilde{P}\right) \leq 0$, we get $E(\tilde{P})-C^{\prime}\left(Q_{*}\right) \geq 0$. Denote the optimal output levels of the regret-neutral firms by $Q_{n}^{*}$. Consequently, we can assert that for regret neutral firm, $Q_{n}^{*} \geq Q_{*}$. The result is summarized in the following proposition:

Proposition 1 The regret-neutral competitive firm will surely produce more than its purely risk-averse counterpart; that is, $Q_{n}^{*}$ is greater than $Q_{*}$.

One may believe that "regret-neutral" is "no regret", and thus, it should be the same as "risk aversion". We note that "regret neutral" is different from "risk aversion". "risk aversion" is when $\beta=0$, and thus, it is "no regret", while "regret-neutral" 
is when " $G$ " $=0$ " which includes the case in which $\beta \neq 0$. We have three cases: (a) "G" $G^{\prime \prime} 0$ " (regret aversion), (b) "G" $=0 "$ (regret neutral), and (c) "G" $<0$ ". Case (c) is not interested, and thus, we do not discuss it in our paper. Since "regret neutral" is when " $G$ " $=0$ " and $\beta$ could be different from zero. Thus, the optimal production of the regret-neutral competitive firm is different from its purely risk-averse counterpart and from Proposition 1, we confirm that the regret-neutral competitive firm will surely produce more than the purely risk-averse firm.

Let $\Pi_{n}^{*}(\tilde{P})=\tilde{P} Q_{n}^{*}-C\left(Q_{n}^{*}\right)$ and $G_{n}(\cdot)$ be the regret function of the regret-neutral firm. For the regret-neutral firm, $G_{n}^{\prime}(\cdot) \equiv m>0$, and hence, from condition (2.3), we obtain:

$$
H_{n}\left(Q_{n}^{*}\right)=\operatorname{Cov}\left(U^{\prime}\left[\Pi_{n}^{*}(\tilde{P})\right], \tilde{P}\right)+E\left\{U^{\prime}\left[\Pi_{n}^{*}(\tilde{P})\right]+\beta m\right\}\left[E(\tilde{P})-C^{\prime}\left(Q_{n}^{*}\right)\right]=0 .
$$

From the above formula, we can get $E(\tilde{P})-C^{\prime}\left(Q_{n}^{*}\right)>0$. Let $Q^{0}$ denote the optimal output level when the uncertain per-unit price, $\tilde{P}$, is fixed at its expected value, $E(\tilde{P})$. For $Q^{0}$, we have $C^{\prime}\left(Q^{0}\right)=E(\tilde{P})$. Thus, we can conclude that the regret-neutral firm produces less than firms under certainty. The result is summarized in the following proposition:

Proposition 2 The regret-neutral competitive firm will surely produce less than firms under certainty; that is, $Q_{n}^{*}$ is smaller than $Q^{0}$.

Combing Proposition 1, we can conclude that $Q^{0}>Q_{n}^{*}>Q_{*}$. For the general regret-averse firm, we cannot assert that the regret-averse competitive firm will surely produce less than the firms under certainty without imposing any condition. In fact, some previous studies, including Wong (2014), Niu et al. (2014), Egozcue et al. (2015) and Broll et al. (2016, 2017), have presented different sufficient conditions to assure that the regret-averse firms produce less than firms under certainty. 


\subsection{Regret-averse competitive firm}

In order to study the behavior of the regret-averse competitive firm, we need to compare the covariance $\operatorname{Cov}\left(G^{\prime}\left[\Pi^{\max }(\tilde{P})-\Pi_{*}(\tilde{P})\right], \tilde{P}\right)$ and the positive value

$$
E\left\{G^{\prime}\left[\Pi^{\max }(\tilde{P})-\Pi_{*}(\tilde{P})\right]\right\}\left[E(P)-C^{\prime}\left(Q_{*}\right)\right]
$$

to generally determine the sign of the term $H\left(Q_{*}\right)$. For this purpose, we first present the following lemma to determine the sign of $\operatorname{Cov}(\phi(\tilde{X}), \tilde{X})$ given $\tilde{X} \in[\underline{X}, \bar{X}]$ and $\phi(\cdot)$ is convex:

Lemma 3 For convex function $\phi(\tilde{X})$ with $\tilde{X} \in[\underline{X}, \bar{X}]$, we have:

1. if $\phi^{\prime}(\underline{X}) \geq 0$, then $\operatorname{Cov}(\phi(\tilde{X}), \tilde{X})>0$;

2. if $\phi^{\prime}(\bar{X}) \leq 0$, then $\operatorname{Cov}(\phi(\tilde{X}), \tilde{X})<0$;

3. if $\phi^{\prime}(\underline{X})<0<\phi^{\prime}(\bar{X})$, and

(a) if $E \phi(\tilde{X}) \geq \phi(\underline{X})$, then $\operatorname{Cov}(\phi(\tilde{X}), \tilde{X})>0$; or

(b) if $E \phi(\tilde{X}) \geq \phi(\bar{X})$, then $\operatorname{Cov}(\phi(\tilde{X}), \tilde{X})<0$.

Determining the sign of the covariance of one random variable and its function is an important problem, with many applications. However, existing studies are mainly designed for completely monotone functions, see, for example, Egozcue, et al. (2009, 2011a, 2011b) and the references therein for more information. However, sometimes, the function studied is not monotone on the entire support. Lemma 3 gives an easy and concrete approach to sign the covariance of one random variable and its convex function discussed in our paper, which enables us to develop properties for the regretaverse competitive firm. Now, we turn back to study the regret-averse firm. We define $\phi(\tilde{P})=G^{\prime}\left[\Pi^{\max }(\tilde{P})-\Pi_{*}(\tilde{P})\right]$. It can be shown that

$$
\begin{aligned}
\phi^{\prime}(\tilde{P}) & =G^{\prime \prime}\left[\Pi^{\max }(\tilde{P})-\Pi_{*}(\tilde{P})\right]\left(Q(\tilde{P})-Q_{*}\right) \\
\phi^{\prime \prime}(\tilde{P}) & =G^{\prime \prime \prime}\left[\Pi^{\max }(\tilde{P})-\Pi_{*}(\tilde{P})\right]\left(Q(\tilde{P})-Q_{*}\right)^{2}+G^{\prime \prime}\left[\Pi^{\max }(\tilde{P})-\Pi_{*}(\tilde{P})\right] Q^{\prime}(\tilde{P}) .
\end{aligned}
$$


Since $Q^{\prime}(\tilde{P})=1 / C^{\prime \prime}(Q(\tilde{P}))>0, G^{\prime \prime}(\cdot)>0$, and if we further assume that $G^{\prime \prime \prime}(\cdot) \geq 0^{4}$, we conclude that $\phi^{\prime \prime}(\cdot)>0$ and $\phi(\cdot)$ is a convex function. As a result, applying Lemma 3 , we obtain the sign of the covariance $\operatorname{Cov}(\phi(\tilde{P}), \tilde{P})$. In addition, recall that $\underline{P}<C^{\prime}\left(Q_{*}\right)<\bar{P}, C^{\prime}[Q(P)]=P$ and $C^{\prime \prime}(\cdot)>0$, Thus, we have $Q(\underline{P})<Q_{*}<Q(\bar{P})$ and $\phi^{\prime}(\underline{P})<0<\phi^{\prime}(\bar{P})$. From the above discussion, we have the following result:

Proposition 4 If $G^{\prime \prime \prime}(\cdot) \geq 0$, the regret-averse competitive firm will produce more than its purely risk-averse counterpart; that is, $Q^{*}$ is greater than $Q_{*}$, when $E G^{\prime}\left[\Pi^{\max }(\tilde{P})-\right.$ $\left.\Pi_{*}(\tilde{P})\right] \geq G^{\prime}\left[\Pi^{\max }(\underline{P})-\Pi_{*}(\underline{P})\right]$.

When the competitive firm is regret-neutral, the condition $E G^{\prime}\left[\Pi^{\max }(\tilde{P})-\Pi_{*}(\tilde{P})\right] \geq$ $G^{\prime}\left[\Pi^{\max }(\underline{P})-\Pi_{*}(\underline{P})\right]$ holds automatically. As a result, Proposition 1 is a special case of Proposition 4, a proposition with more general result.

The intuition for Proposition 4 is as follows: If $E G^{\prime}\left[\Pi^{\max }(\tilde{P})-\Pi_{*}(\tilde{P})\right] \geq G^{\prime}\left[\Pi^{\max }(\underline{P})-\right.$ $\left.\Pi_{*}(\underline{P})\right]$, it follows from Lemma 3 that $G^{\prime}\left[\Pi^{\max }(\tilde{P})\right]$ is positively correlated with $\tilde{P}$. Introducing regret-aversion to the firm makes the firm raise more concern about the disutility from the discrepancy of its output level, $Q(\tilde{P})-Q_{*}$, when high realizations of $\tilde{P}$ are revealed. To minimize regret, the regret-averse firm optimally adjusts its output level upward from $Q_{*}$ to $Q^{*}$ with $Q^{*}>Q_{*}$.

We note that the above condition is sufficient, but not necessary. This leads to the following property.

Property 5 As long as

$$
\operatorname{Cov}\left(G^{\prime}\left[\Pi^{\max }(\tilde{P})-\Pi_{*}(\tilde{P})\right], \tilde{P}\right) \geq-E\left\{G^{\prime}\left[\Pi^{\max }(\tilde{P})-\Pi_{*}(\tilde{P})\right]\right\}\left[E(P)-C^{\prime}\left(Q_{*}\right)\right]
$$

we have $Q^{*}>Q_{*}$. This implies that negative correlation between $G^{\prime}\left[\Pi^{\max }(\tilde{P})-\Pi_{*}(\tilde{P})\right]$ and $\tilde{P}$ is allowed, as long as they are not too negatively correlated. As shown before,

\footnotetext{
${ }^{4}$ This assumption has been made by many authors, see for instance Wong (2014), Niu et al. (2014) and Broll et al. (2016, 2017).
} 
whenever $E_{\Phi}(\tilde{P}) \geq C^{\prime}\left(Q_{*}\right)$, the regret averse firm would produce more than purely risk-averse firm.

Broll et al. (2017) present sufficient conditions under which, the regret-averse firms produce more than firms without uncertainty. Recall that $Q^{0}$ is the optimal output level under certainty. Since $E(\tilde{P})>C^{\prime}\left(Q_{*}\right)$ and $C^{\prime \prime}(\cdot) \geq 0$, we have $Q^{0}>Q_{*}$. Then, under conditions designed by Broll et al. (2017), we can also get $Q^{*}>Q^{0}>Q_{*}$. However, to get $Q^{*}>Q^{0}$, Broll et al. (2017) not only require $E G^{\prime}\left[\Pi^{\max }(\tilde{P})-\Pi^{0}(\tilde{P})\right] \geq$ $G^{\prime}\left[\Pi^{\max }(\underline{P})-\Pi^{0}(\underline{P})\right]$, they also set the regret coefficient $\beta \geq \beta_{0}>0$. Here, $\Pi^{0}(\tilde{P})=$ $\tilde{P} Q^{0}-C\left(Q^{0}\right)$ and $\beta_{0}$ is a specified value. In this paper, we directly compare $Q^{*}$ and $Q_{*}$, and thus, we allow the regret coefficient $\beta$ to be any positive value; that is, under condition $E G^{\prime}\left[\Pi^{\max }(\tilde{P})-\Pi_{*}(\tilde{P})\right] \geq G^{\prime}\left[\Pi^{\max }(\underline{P})-\Pi_{*}(\underline{P})\right]$, any regret-averse firm will produce more than its purely risk-averse counterpart, even its regret coefficient $\beta$ is very small.

In the above, we focus on the comparison of the optimal output levels of firms with $\beta>0$ (regret-averse firms) and $\beta=0$ (purely risk-averse firms). In the following, we turn to compare the optimal output levels of two firms with two different positive $\beta$ 's. To this end, we study the comparative statics of the optimal output levels when the regret coefficient $\beta$ varies as shown in the following proposition:

Proposition 6 If $G^{\prime \prime \prime}(\cdot) \geq 0$, the regret-averse firm's optimal output level, $Q^{*}$, satisfies the following: if $E G^{\prime}\left[\Pi^{\max }(\tilde{P})-\Pi_{*}(\tilde{P})\right] \geq G^{\prime}\left[\Pi^{\max }(\underline{P})-\Pi_{*}(\underline{P})\right]$, then $Q^{*}$ will surely increase with an increase in the regret coefficient $\beta$.

One could interpret the regret coefficient $\beta$ as "if the firm's regret coefficient $\beta$ is larger, then the firm is more regret-averse." Thus, from Proposition 6, we get the following property:

Property 7 If $G^{\prime \prime \prime}(\cdot) \geq 0$ and $E G^{\prime}\left[\Pi^{\max }(\tilde{P})-\Pi_{*}(\tilde{P})\right] \geq G^{\prime}\left[\Pi^{\max }(\underline{P})-\Pi_{*}(\underline{P})\right]$, then if the firm is more regret-averse, then it will produce more. 


\subsection{Regret-neutral firm versus regret-averse firm}

In the following, we compare the optimal output levels between the regret-neutral and regret-averse firms. To fully evaluate the effect of regret function, we assume the von Neumann-Morgenstern utility function $U(\cdot)$ is the same. Recall that the optimal output levels of the regret-averse firms and regret-neutral firms are denoted by $Q^{*}$ and $Q_{n}^{*}$, respectively. We have:

$$
\begin{gathered}
H\left(Q^{*}\right)=E\left\{\left\{U^{\prime}\left[\Pi^{*}(\tilde{P})\right]+\beta G^{\prime}\left[\Pi^{\max }(\tilde{P})-\Pi^{*}(\tilde{P})\right]\right\}\left[\tilde{P}-C^{\prime}\left(Q^{*}\right)\right]\right\}=0, \\
H_{n}\left(Q_{n}^{*}\right)=E\left\{\left\{U^{\prime}\left[\Pi_{n}^{*}(\tilde{P})\right]+\beta G_{n}^{\prime}\left[\Pi^{\max }(\tilde{P})-\Pi_{n}^{*}(\tilde{P})\right]\right\}\left[\tilde{P}-C^{\prime}\left(Q_{n}^{*}\right)\right]\right\}=0,
\end{gathered}
$$

where $\Pi_{n}^{*}(\tilde{P})=\tilde{P} Q_{n}^{*}-C\left(Q_{n}^{*}\right)$ and $G_{n}(\cdot)$ is the regret function of regret-neutral firm. Evaluating $H(\cdot)$ at $Q_{n}^{*}$, we have

$$
\begin{aligned}
H\left(Q_{n}^{*}\right)= & E\left\{\left\{U^{\prime}\left[\Pi_{n}^{*}(\tilde{P})\right]+\beta G^{\prime}\left[\Pi^{\max }(\tilde{P})-\Pi_{n}^{*}(\tilde{P})\right]\right\}\left[\tilde{P}-C^{\prime}\left(Q_{n}^{*}\right)\right]\right\} \\
= & \beta E\left\{\left\{G^{\prime}\left[\Pi^{\max }(\tilde{P})-\Pi_{n}^{*}(\tilde{P})\right]-G_{n}^{\prime}\left[\Pi^{\max }(\tilde{P})-\Pi_{n}^{*}(\tilde{P})\right]\right\}\left[\tilde{P}-C^{\prime}\left(Q_{n}^{*}\right)\right]\right\} \\
= & \beta \operatorname{Cov}\left(G^{\prime}\left[\Pi^{\max }(\tilde{P})-\Pi_{n}^{*}(\tilde{P})\right], \tilde{P}\right)+\beta E\left\{G^{\prime}\left[\Pi^{\max }(\tilde{P})-\Pi_{n}^{*}(\tilde{P})\right]-m\right\} \\
& \times\left[E(\tilde{P})-C^{\prime}\left(Q_{n}^{*}\right)\right] .
\end{aligned}
$$

Then according to the first and second order conditions, we would have $Q_{n}^{*} \leq(\geq$ )$Q^{*}$ if $H\left(Q_{n}^{*}\right) \geq(\leq) 0$. From Proposition 2, we know that a sufficient condition for $H\left(Q_{n}^{*}\right) \geq(\leq) 0$ is as follows:

$$
\begin{aligned}
& \operatorname{Cov}\left(G^{\prime}\left[\Pi^{\max }(\tilde{P})-\Pi_{n}^{*}(\tilde{P})\right], \tilde{P}\right) \geq(\leq) 0 ; \\
& E G^{\prime}\left[\Pi^{\max }(\tilde{P})-\Pi_{n}^{*}(\tilde{P})\right] \geq(\leq) m .
\end{aligned}
$$

Consequently, from Lemma 3 and Proposition 4, we have the following Proposition:

Proposition 8 If $G^{\prime \prime \prime}(\cdot) \geq 0$, the regret-averse competitive firm will produce more (or less) than its regret-neutral counterpart; that is, $Q^{*}$ is greater (or smaller) than $Q_{n}^{*}$, when $E G^{\prime}\left[\Pi^{\max }(\tilde{P})-\Pi_{n}^{*}(\tilde{P})\right] \geq(\leq) G^{\prime}\left[\Pi^{\max }(\underline{P})-\Pi_{n}^{*}(\underline{P})\right]$ and $E G^{\prime}\left[\Pi^{\max }(\tilde{P})-\right.$ $\left.\Pi_{n}^{*}(\tilde{P})\right] \geq(\leq) m$ 


\section{A binary model}

To get more insight, in this section we analyze a simple binary model in which $\tilde{P}$ takes on the low value, $\underline{P}$, with probability $q$ and the high value, $\bar{P}$, with probability $1-q$, where $0<q<1$. In such a binary model, the right-hand sides of Equations (2.3) and (2.4) become

$$
\begin{aligned}
& q\left\{U^{\prime}\left[\Pi^{*}(\underline{P})\right]+\beta G^{\prime}\left[\Pi^{\max }(\underline{P})-\Pi^{*}(\underline{P})\right]\right\}\left[\underline{P}-C^{\prime}\left(Q^{*}\right)\right] \\
& +(1-q)\left\{U^{\prime}\left[\Pi^{*}(\bar{P})\right]+\beta G^{\prime}\left[\Pi^{\max }(\bar{P})-\Pi^{*}(\bar{P})\right]\right\}\left[\bar{P}-C^{\prime}\left(Q^{*}\right)\right]=0 .
\end{aligned}
$$

and

$$
q U^{\prime}\left[\Pi_{*}(\underline{P})\right]\left[\underline{P}-C^{\prime}\left(Q_{*}\right)\right]+(1-q) U^{\prime}\left[\Pi_{*}(\bar{P})\right]\left[\bar{P}-C^{\prime}\left(Q_{*}\right)\right]=0 .
$$

We define the following threshold value:

$$
q^{+}=\left\{1-\frac{U^{\prime}\left[\underline{P} Q^{+}-C\left(Q^{+}\right)\right]\left[\underline{P}-C^{\prime}\left(Q^{+}\right)\right]}{U^{\prime}\left[\bar{P} Q^{+}-C\left(Q^{+}\right)\right]\left[\bar{P}-C^{\prime}\left(Q^{+}\right)\right]}\right\}^{-1}
$$

where $Q^{+}$is the quantity of output that solves

$$
\Pi^{\max }(\bar{P})-\left[\bar{P} Q^{+}-C\left(Q^{+}\right)\right]=\Pi^{\max }(\underline{P})-\left[\underline{P} Q^{+}-C\left(Q^{+}\right)\right]
$$

Comparing $Q^{*}$ and $Q_{*}$ yields the following proposition.

Proposition 9 In the binary model, if $G^{\prime \prime \prime}(\cdot) \geq 0$, the regret-averse firm produces more than the purely risk-averse firm; that is, $Q^{*}>Q_{*}$, if the probability that $\tilde{P}=\underline{P}$ is above the critical value, $q^{+}$which is defined in (3.7).

To see the intuition underlying Proposition 9, we first suppose that the firm is purely risk-averse. In this situation, when the realization of the low price is more likely, the firm will optimally produce less so as to minimize the variability of its profit at date 1 . Thus, the optimal output level, $Q_{*}$, is close to $Q(\underline{P})$ and further away from $Q(\bar{P})$, where the former is ex-post optimal when $\tilde{P}=\underline{P}$, and the latter 
is ex-post optimal when $\tilde{P}=\bar{P}$. Including regret-aversion in the objective function makes the firm take into account the disutility from the discrepancy of the output level $Q(\bar{P})-Q_{*}$, should the actual price be high, i.e., $\tilde{P}=\bar{P}$. To avoid regret, the firm is induced to produce more so that $Q^{*}>Q_{*}$.

From Proposition 6, we can easily get the following result:

Proposition 10 In the binary model, if $G^{\prime \prime \prime}(\cdot) \geq 0$, the regret-averse firm will produce more if the firm is more regret-averse; that is, $d Q^{*} / d \beta>0$, if the probability that $\tilde{P}=\underline{P}$ is above the critical value $q^{+}$which is defined in (3.7).

In our paper we set the condition $G^{\prime \prime \prime}(\cdot) \geq 0$ in most, if not all, propositions. We note that in our paper we do not consider the situation in which $G^{\prime \prime \prime}(\cdot)<0$. It is because the function $\phi(\cdot)$ would not be convex if $G^{\prime \prime \prime}(\cdot)<0$, and hence, we cannot use Lemma 3. This makes the comparison of $Q^{*}$ and $Q_{*}$ unclear. Thus, we do not consider this situation in our paper. We also note that the condition $E G^{\prime}\left[\Pi^{\max }(\tilde{P})-\Pi_{*}(\tilde{P})\right] \geq G^{\prime}\left[\Pi^{\max }(\underline{P})-\Pi_{*}(\underline{P})\right]$ plays a key role in our paper to compare the optimal outputs of regret-averse and purely risk-averse firms. It is now clear that in the binary model, when the probability that $\tilde{P}=\underline{P}$ is below the critical value $q^{+}$, the condition $E G^{\prime}\left[\Pi^{\max }(\tilde{P})-\Pi_{*}(\tilde{P})\right] \geq G^{\prime}\left[\Pi^{\max }(\underline{P})-\Pi_{*}(\underline{P})\right]$ could fail. However, kindly note that this condition is a sufficient condition for $Q^{*} \geq Q_{*}$ but not necessary. Thus even $E G^{\prime}\left[\Pi^{\max }(\tilde{P})-\Pi_{*}(\tilde{P})\right]<G^{\prime}\left[\Pi^{\max }(\underline{P})-\Pi_{*}(\underline{P})\right]$ hold, we may not have $Q^{*}<Q_{*}$. Readers may read Property 5 for more information. We note that in Property 5 , the situation that $G^{\prime}\left[\Pi^{\max }(\tilde{P})-\Pi_{*}(\tilde{P})\right]$ and $\tilde{P}$ is too negatively correlated will not happen in most empirical situations. Thus, we make the follow conjecture in the paper:

Conjecture 11 Except the situation that $G^{\prime}\left[\Pi^{\max }(\tilde{P})-\Pi_{*}(\tilde{P})\right]$ and $\tilde{P}$ is too negatively correlated (this situation will not happen in most empirical situations), the regret-averse firms will always produce more than risk-averse counterparts. 
We also make the following conjecture:

Conjecture 12 In general, if the firm is more regret-averse, then it will produce more.

\section{Conclusion}

Some authors, such as Wong (2014), Niu et al. (2014), Egozcue et al. (2015), and Broll et al. (2016, 2017), have investigated several sufficient conditions under which the regret-averse firms will produce less than firms under certainty. In this paper, we extend their studies to develop more properties not only for the regret-averse firms, but also for the regret-neutral firms. We compare the optimal output levels among regret-neutral, regret-averse, and purely risk-averse firms under uncertainty and firms under certainty. We first show that different from the findings in the literature to show that the regret-averse firms produce less than firms under certainty only under some sufficient conditions, the regret-neutral competitive firm will surely produce less than firms under certainty without imposing any condition. We also show that the regret-neutral competitive firm will surely produce more than its purely risk-averse counterpart.

Thereafter, we show how to determine the sign of the covariance between a random variable and its convex function in any general case. This property enables us to derive the properties for the regret-averse firms. We show that under some conditions, more regret-averse firms will produce more outputs and the regret-averse competitive firm will produce more than both purely risk-averse and regret-neutral counterparts.

Last, we set the uncertain output price to take either low or high values with positive probability. Under this simple binary model, we find the possibility that the regret-averse firm may optimally produce more than purely risk-averse firm, especially when the low output price is very likely to prevail. This weakens the conditions set in the general case for the regret-averse competitive firm to produce more than its 
purely risk-averse counterpart or when the firm is more regret-averse. Thus, we set two conjectures in this paper: in general, (1) the regret-averse firms will always produce more than risk-averse firms, and (2) if the firm is more regret-averse, then it will produce more.

Together with the existing findings in the literature, the findings in this paper make a clear picture about the optimal production decisions of both regret-neutral and regret-averse firms. Our findings could then be useful for production managers in their decision on the production.

\section{References}

1. Bell, D. E. (1982), Regret in decision making under uncertainty. Operations Research, 30, 961-981.

2. Braun, M., Muermann, A. (2004), The impact of regret on the demand for insurance. Journal of Risk and Insurance, 71, 737-767.

3. Broll, U. (1992), The effect of forward markets on multinational firms. Bulletin of Economic Research, 44, 233-240.

4. Broll, U., Welzel, P., Wong, K. P. (2016), Regret theory and the competitive firm revisited, Eurasian Economic Review, 6, 481-487.

5. Broll, U., Welzel, P., Wong, K. P. (2017), The firm under regret aversion. Working paper.

6. Egozcue, M., Fuentes García, L., Wong, W. K. (2009), On some covariance inequalities for monotonic and non-monotonic functions, Journal of Inequalities in Pure and Applied Mathematics, 10(3), Article 75, 1-7. 
7. Egozcue, M., Fuentes García, L., Wong, W. K., Zitikis, R. (2011a), Grüss-type bounds for covariances and the notion of quadrant dependence in expectation, Central European Journal of Mathematics, 9(6), 1288-1297.

8. Egozcue, M., Fuentes García, L., Wong, W. K., Zitikis, R. (2011b), The covariance sign of transformed random variables with applications to economics and finance, IMA Journal of Management Mathematics, 22(3), 291-300.

9. Egozcue, M., Guo, X., Wong, W. K. (2015), Optimal output for the regret-averse competitive firm under price uncertainty, Eurasian Economic Review, 5, 279-295.

10. Loomes, G. (1988), Further evidence of the impact of regret and disappointment in choice under uncertainty. Economica, 55, 47-62.

11. Loomes, G., Sugden, R. (1982), Regret theory: an alternative theory of rational choice under uncertainty. Economic Journal, 92, 805-824.

12. Loomes, G., Sugden, R. (1987), Testing for regret and disappointment in choice under uncertainty. Economic Journal, 97, 118-129.

13. Muermann, A., Mitchell, O. S., Volkman, J. M. (2006), Regret, portfolio choice and guarantees in defined contribution schemes. Insurance: Mathematics and Economics, 39, 219-229.

14. Niu, C. Z., Guo, X., Wang, T., Xu, P. R. (2014), Regret theory and the competitive firm: a comment, Economic Modelling, 41, 313-315.

15. Quiggin, J. (1994), Regret theory with general choice sets. Journal of Risk and Uncertainty, 8, 153-165.

16. Sandmo, A. (1971), On the theory of the competitive firm under price uncertainty. American Economic Review, 61, 65-73. 
17. Starmer, C., Sugden, R. (1993), Testing for juxtaposition and event-splitting effects. Journal of Risk and Uncertainty, 6, 235-254.

18. Sugden, R. (1993), An axiomatic foundation of regret. Journal of Economic Theory, 60, 159-180.

19. Viaene, J. M., Zilcha, I. (1998), The behavior of competitive exporting firms under multiple uncertainty. International Economic Review, 39, 591-609.

20. Wong, K. P. (2014), Regret theory and the competitive firm. Economic Modelling, 36, $172-175$. 